\title{
Risk-based EMC Approach in Hospital Environment
}

\author{
Mumpy Das \\ University of Twente \\ Enschede, The Netherlands \\ mumpy.das@utwente.nl
}

\author{
Robert Vogt-Ardatjew \\ University of Twente \\ Enschede, The Netherlands
}

\author{
Bärbel van den Berg \\ MST Hospital \\ Enschede, The Netherlands
}

\author{
Frank Leferink \\ University of Twente, Enschede \\ Thales Nederland, Hengelo
}

\begin{abstract}
A modern hospital has a wide range of medical devices that can be technologically very advanced and complex in terms of electromagnetic emissions and susceptibility. It is a public environment where people carry communication equipment and/or medical active implants. Therefore, electromagnetic interference among the devices in the hospital environment is not an unfamiliar topic. There is a misconception among engineers that if all medical devices comply with harmonized standards, then the installation of a large number of medical devices altogether would work absolutely fine. However, this is not always true, and so for complex systems, a smarter approach is necessary to assess and control the risks of electromagnetic interference. This paper emphasizes the importance of using the risk-based approach to deal with the often unpredictable factors causing electromagnetic interference in a complex hospital environment.
\end{abstract}

Keywords-electromagnetic compatibility, hospital, rule-based EMC, risk-based EMC

\section{INTRODUCTION}

Nowadays, medical devices (MD) used in hospitals are more advanced than those of the ancient days as they are being used for electromagnetic (EM) vehicle and communication purposes as well. It is only less than a decade ago that visitors were not allowed to bring or use any mobile communication device to a hospital. Although most of the MD are advanced, there are some MD from that era that and not yet replaced due to convenience or budget. New equipment is tested using electromagnetic compatibility (EMC) standards [1] according to the rule-based EMC approach to avoid the chance of electromagnetic interference (EMI) at initial stage before installing any equipment in the hospital. The minimum immunity requirements of medical devices are set by the international standard IEC 60601-1-2 [1], although also nonmedical devices are present in hospitals. The main intention as an engineer is not only to test the MD by applying the harmonized standards but also to observe and make sure that the MD is working properly even under circumstances of high EM field and thus satisfy rules for EMC. This is actually what is written in the Law related to EMC, i.e. the EMC Directive

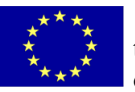

The research leading to these results has received funding from the European Union's Horizon 2020 research and innovation programme under the Marie Skłodowska-Curie Grant Agreement No 812.790 (MSCA-ETN PETER). This publication reflects only the authors view, exempting the European Union from any liability. Project website: http://etn-peter.eu/
(EMCD) [2]: "Equipment shall be so designed and manufactured, having regard to the state of the art, as to ensure that: it has a level of immunity to the electromagnetic disturbance to be expected in its intended use which allows it to operate without unacceptable degradation of its intended use". The newest Guide on EMC [2] as well as the 4th edition of IEC 60601-1-2 [3] advocates the risk-based approach instead of the usual rule-based approach. This approach is not new. It includes four essential steps that have been discussed in Section IV below. The steps include the assessment of the risk of the victims when exposed to the sources. With the help of a sourcevictim matrix, it is possible to assess the most vulnerable victims of the sources present in that environment. As a final step validation and verification can be done to get the actual results as compared to the result after following IEC60601-1-2 standard. This method has been used for decades in the defense electronics world [4] and has recently been implemented by Lloyd's as an alternative to the conventional rule-based approach for ships [5]. From both cost and legal perspective, this approach outperforms the rule-based approach.

In a hospital environment, many sources are present that generate EM emissions both intentionally and unintentionally [6]. Intentional sources produce EM fields for patient imaging, e.g. magnetic resonance imaging (MRI), or therapy, e.g. radio frequency (RF) surgery. In addition, many types of MD use EM fields for mobile and radio communication [7]. Also, security devices like metal detectors can be present in hospitals. Strong intentional sources like MRI and RF surgery can cause EMI in hospital environment [8]. The introduction of TETRA communication equipment for ambulance personnel caused a series of unexpected interference cases [9] and it was shown that 5 out of $14 \mathrm{MD}$ were sensitive to the TETRA radio. In another case study, it was shown that during breast surgery the usage of the RF electrosurgery generator stopped patient's pacemaker in the hospital [10], while a pulse oximeter stopped working due to the placement of a two-way radio nearby.

In Medisch Spectrum Twente (MST) hospital, a device for monitoring the neonatal, cardiotocography (CTG), showed a clinically relevant output signal, even without the presence of a child, due to the placement of a Wi-Fi router nearby, as shown in Fig.1. Furthermore, various articles showed that mobile phones, walkie-talkie, or other wireless communication equipment created interference in the implanted pacemaker[11] 


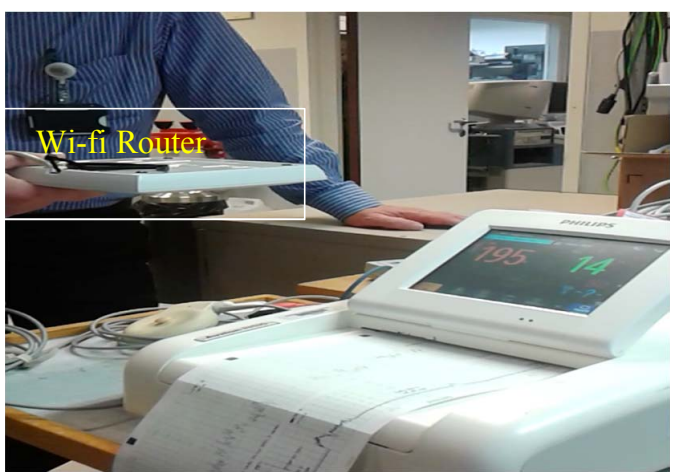

Fig. 1. The real-life example of EMI (CTG interference)

and some malfunctions in 4/7 infusion pumps and 1/4 syringe pumps [12]. These examples and ongoing incidents are quite worrying about the needs of EMC in the hospital environment. On the other hand, unintentional EM field sources are those where EM energy is emitted as a side effect of the normal functionality, for example, LED lights, electronically operated doors, leakage of microwave ovens, etc. [6]. It is not uncommon for devices to generate several types of emissions at the same time. But also uncontrolled unintentional interference caused by wireless devices of visitors or ignorant staff, or external personal carrying out maintenance (drilling or welding equipment) are apparent in the modern hospital environment.

The 4th edition of IEC 60601-1-2 [3] focuses more on risk analysis, moves away from equipment categories, and considers "intended use environments" by defining professional healthcare, home healthcare, and special environments. Although the use of IEC 60601-1-2 [3] is mandatory since $31^{\text {st }}$ December 2018, as mentioned in [13], to meet the new medical device regulation (MDR) the medical device will have three years of transition time. In the regulation (EU) 2017/745 [14] it is mentioned that "The common specifications for each of the groups of products listed in Annex XVI shall address, at least, application of risk management as set out in Annex I for the group of products in question and, where necessary, clinical evaluation regarding safety". Furthermore, the organizations responsible for the intended use environment would also like to fulfill this new risk-based approach. For hospitals, the risk-based approach will be an essential tool for assessing their medical workspaces as the electromagnetic field environment is getting more and more complex as well as dynamically changing in space and time. This paper gives a short overview of known interference cases in the hospital environment showing the need to control EMC. Then, we discuss the behavior of MD as possible sources of the EMI in hospitals. The difference between the rule-based EMC approach and risk-based EMC approach is discussed. As hospital environments are semi-enclosed with light cavity resonance effects, we have performed experiments to show a graphical representation of the EM field distribution.

\section{FACTORS LINKED TO EMI IN HOSPITALS}

The environment, location, and behavior of MD and other (non-medical) devices play a vital role in EMC. Various papers show measurements of EM fields in hospitals [15], [16], [17] justifying the importance of the environmental influence, implying that the risk-based approach is more practical than the rule-based approach. It is very important to keep in mind that the environment in the hospital is much more complex than the free space because a hospital can behave like a semireverberant environment [18], [19]. The location of the sources changes unpredictably over time as people in the hospital carry mobile devices, electric cars move through the corridors 24/7, elevators move from floor to floor [20], antennas are placed in different positions inside or outside of the building, routers are present inside rooms, etc. This further increases the complexity of the fields within such a place.

\section{A. Sources of EMI}

There is an exponential growth of the need of wireless communication in MD and researchers are mainly focused to propose some low cost and low power MD [21] EMI in the hospital environments can be created by various wireless connections like Bluetooth, Wi-Fi/WLAN, etc., which is discussed in [22], [23], and [24]. These wireless connections are used in various devices like phones, headphones, laptops, tablets, ambulance, fax machines, and many more. WLAN operating at $2.45 \mathrm{GHz}$, with $100 \mathrm{~mW}$ adapters creates interference with other MD if the distance of separation is less than $10 \mathrm{~cm}$ [23]. As mentioned in [25], the installation of the Internet of Things (IoT) in hospitals will save the time of the staff, be beneficial to patients, and will upgrade the quality of treatment, but will increase EMI risks. Light-emitting diode (LED) lights are mostly used in modern hospitals as they are energy-saving and can be used longer than conventional lights, but it has been shown that LED is one of the causes for generating EMI [26]. Uses of RF electrosurgical units (ESU) in operation theatre may also lead to EMI with other MD [27]. Radiofrequency identification (RFID) has become very popular in the hospital to enhance safety, add convenience and reduce the cost of health care, but again, it increases the risk of interference created by RFID on MD and vice-versa [28].

\section{B. Victims of EMI}

Examples of a victim of EM fields are the nuclear magnetic resonance devices that are used to determine the content and purity of the molecule of a substance. They are highly vulnerable to EMI in hospitals [29]. In [20] it is shown that EMI generated from the elevator in the hospital can impact the implantable cardiac pacemakers inside a human body.

\section{Hospital as a semi-reverberant environment}

The environment can have a strong impact on creating EMI. In a hospital, there are many different materials made of metal like reflecting doors, windows, floor, walls (concrete, coated glass) [25], ceiling, pipes, cables, etc. The EM waves tend to reflect from those structures in multiple directions, changing polarizations, and creating resonances. The outdoor environment is more open and very different from the indoor environment, resulting in fewer and weaker reflections of waves compared to the indoor environment. Most conventional standards are assuming free-space test techniques equivalent to 
the outdoor environment. Depending on the geometry, volume, and materials used within such a semi-reverberant environment like the hospital, the waves reflected within can create resonances, and therefore locally increase the amplitude of the field intensity. Although the fields are distributed similarly to the ones in a classical reverberation chamber (RC), their behavior is not as predictable, even statistically. The decreased field uniformity and isotropy, with respect to a classical RC, due to the inconsistent placement of apertures such as windows and reflectors formed by conductive objects can cause strong variations in the predictions of potential EMI risks.

\section{Behavior}

EM behavior of devices as well as their location and immediate environment plays a very important role in EMI. Before defining the risk-based EMI we also need to understand the behavior of humans, movement of sources, location, number of sources etc. Most of the devices using wireless communication do not transmit continuously and at the same time. The placement of antennas and the unwanted sources affects the field distribution, especially in a semi-reverberant environment, by exciting its different modes. The field intensity along the line of sight is also strongly related to the distance from the transmitter to the victim.

Now, the main question comes into the picture: even though all the devices had gone through certain EMC tests before installation and follow the standard requirements, then why are there still EMI issues in hospitals? We can conclude that the behavior of the device in certain environment plays a vital role here. It adds another layer of complexity that is not taken into account during the aforementioned laboratory tests.

\section{EXPERIMENTAL ENVIRONMENT ANALYSIS}

To get the general idea of the EM field distribution in the hospital environment compared to the free-space environment, we performed experiments with the help of our own received signal strength indicator (RSSI) device. Various authors have used some measurement techniques to examine the EM field distribution in a hospital-like environment. For instance, St. Francis Hospital was using a spectrum analyzer (SA) to measure the EM environment within the frequency range of 30 $\mathrm{MHz}-7 \mathrm{GHz}$ [15]. An EM field meter was used to measure the field intensity within a frequency range of $30 \mathrm{MHz}-3 \mathrm{GHz}$ in various hospitals [16], [17]. Here we used an RSSI device as shown in Fig.2(a), which is a more simple but more representative method to illustrate the distribution of the field in a semi-reverberant environment, such as a hospital. This compact device is made of an Arduino Microcontroller, ESP8266 (Wi-fi module) with a strip of LEDs and an LCD. It has its own Wi-Fi source as shown in Fig.2(b), which is made of ESP8266, microcontroller, and a battery. The strip of LED lights up in green when there is an electromagnetic field around the RSSI device. The RSSI device used here is calibrated to display the power received coming from the $\mathrm{Wi}$-Fi source. It is only used to help visualize the behavior of the fields in various environments as a function of space. We placed the $\mathrm{Wi}-\mathrm{Fi}$ moduleESP8266 operating at $2.4 \mathrm{GHz}$ in one point and walked $2.5 \mathrm{~m}$ away from it with the RSSI device, while making a long

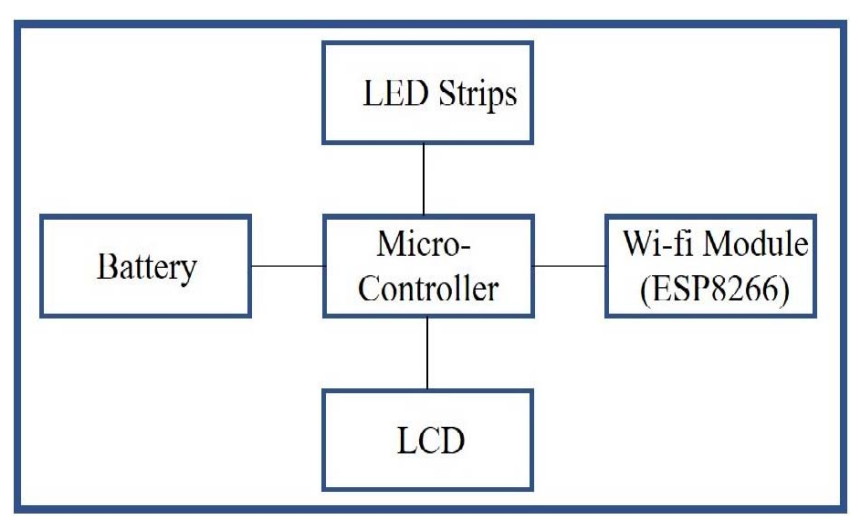

(a)

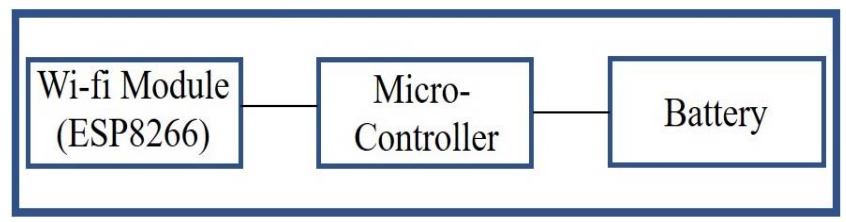

(b)

Fig. 2. (a) RSSI device, (b) Wi-fi source

exposure photo to get an idea about the EM field distribution. Fig3(i) shows the pictorial view of the RSSI device and (ii) the Wi-Fi source. We conducted the experiment in a laboratory and in an anechoic chamber.

Fig.4. shows the field distribution in the anechoic chamber. The number of green LED lights indicates the power received. We can see that in free space there is an exponential decrement of EM field intensity while moving away from the source, with some small deviations. Those deviations are caused by the imperfections of the chamber causing minor reflections e.g. from an uncovered floor, metal ventilation shafts. In Fig.5 we can see a major change in the long exposure shot of the RSSI device inside a lab, containing furniture and metal objects. This room represents a hospital and acts as a semi reverberant environment. We can notice due to the presence of reflectors in the room the EM field is different than the previous result. The summation of the many waves reverberating within this room causes the creation of hotter and colder spots. The power received in the hot spots can be much higher than in free space. Furthermore, even though the position from the source was maintained as in the previous experiment, the power received is not strongly affected by this distance, meaning that the reflected waves are dominant over the line of sight component.

This measurement shows that the EM distribution in the hospital is different from free space due to the reflections. The modification of the field in a reverberant environment might cause an unforeseen increase of the effective field strength with respect to the free space laboratory conditions, coupled onto the victim devices [19], which is not predicted by the classical standards using a rule-based approach. Therefore, this additional variation might be taken into account using the riskbased approach. 


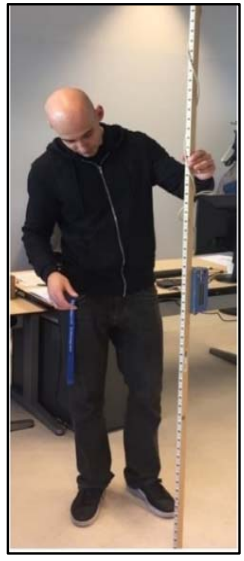

(a)

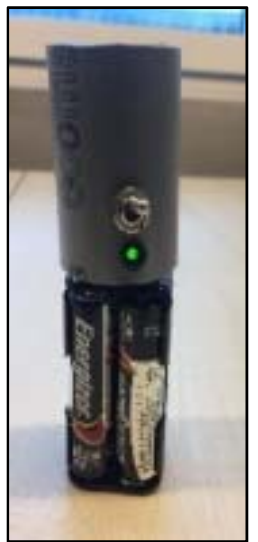

(b)
Fig. 3. (a) Picture of RSSI device (b) Wi-fi source

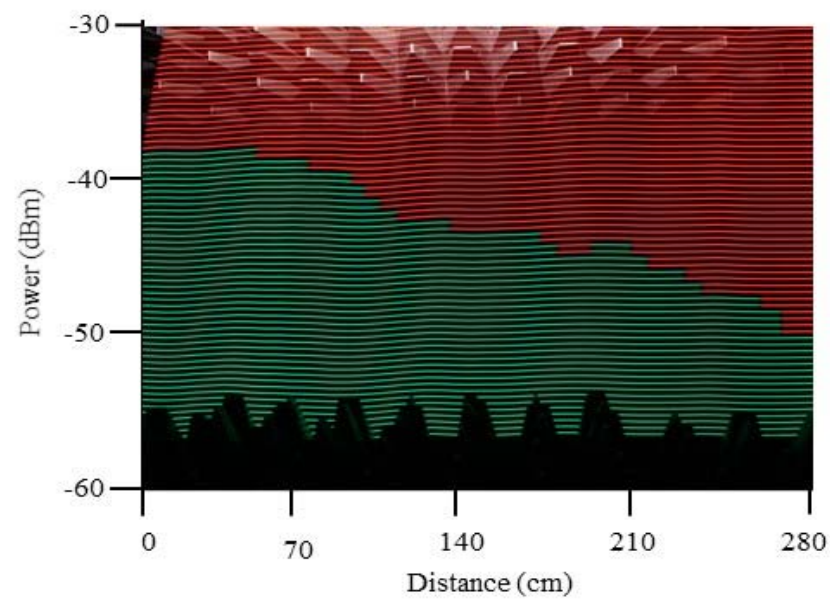

Fig. 4. Long exposure photo of the electro-magnetic field in an anechoic chamber

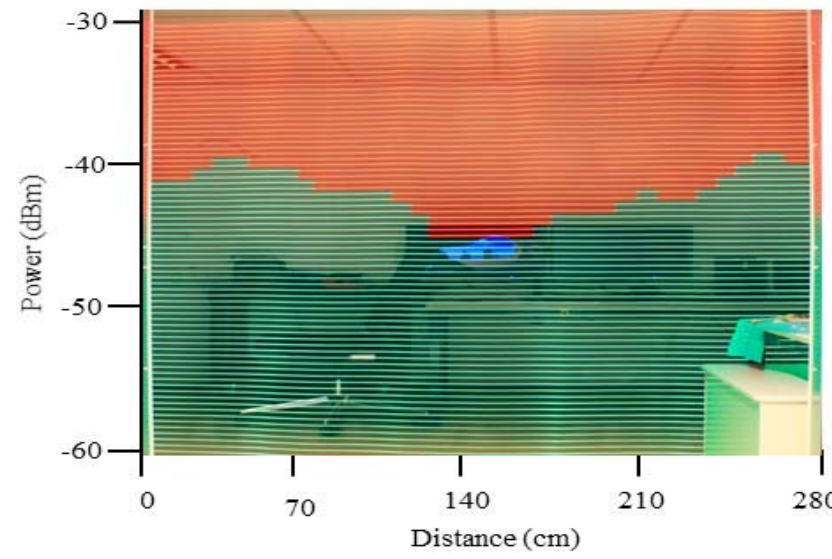

Fig. 5. Long exposure photo of the electro-magnetic field inside a building or hospital.

As opposed to the rule-based approach, the risk-based approach utilizes different parameters to assess the risks of EMI in the given environment. The parameters include the characterization of how severe an effect of a malfunctioning victim MD would have on its patient as well as the probability of such an occurrence depending on the accessibility and availability of the sources. The effect of the environment itself is also taken into consideration. With the help of these parameters, the determination of the high-risk cases is possible when they are plotted in the source-victim matrix [30]. From this matrix, the highest risk cases can be selected, analyzed more closely, as well as validated experimentally.

\section{RULE-BASED AND RISK-BASED EMC APPROACH IN A HOSPITAL ENVIRONMENT}

The rule-based approach was the most common way to determine EMC and it implies mainly following EMI standards. It is easy to apply a rule-based approach for a single MD, but it is difficult to do the same for complex installations with multiple sources. It is difficult to test all parts of MD using harmonized standards. Moreover, even if all the devices present comply with the standards and they have connected together then the set of devices may not fulfill the requirements as needed. Thus, to assess the EM risks in a complex MD we need to follow the risk-based instead of the rule-based approach. In the newest Guide on the European EMC Directive, also a riskbased approach is mentioned. The risk-based approach is already applied for other complex systems such as used in the military domain and for naval ships [5].

The Guide on the EMCD [2] introduced the risk-based approach in December 2018 and requires a risk analysis and a risk assessment. The result of the assessment is then used for performing validation and verification tests. Compliance with the MDR for MD is checked by inspection of the test report and the risk management file. The risk management is carried out to identify the hazards associated with medical devices, to estimate and evaluate the associated risks, to control these risks, and to monitor the effectiveness of these controls throughout the life-cycle of a medical device. Unacceptable risks occur when a product failure causes harm to a patient, operator, or the environment. Risk arises due to a possible hazard causing a possible harm and is defined by the severity of the harm (effect) times the occurrence probability. For any MD the four steps to be taken in a EMC risk assessment are:

1. Electromagnetic risk analysis

2. Electromagnetic risk evaluation

3. Electromagnetic risk control

4. Electromagnetic risk verification and validation

The similarities with the earlier described risk-based approaches are large. However, the Guide for the EMCD [2] and the medical device regulation (MDR) [13] are focusing on the individual devices. As the hospital is a complex environment with many MD installed, the initial step of the risk-based EMC approach is to define the total EM environment of the hospital where the MD are installed. These detailed analyses shall be used to evaluate the risk, and define possible mitigations in the EM control phase. 


\section{CONCLUSION}

Risk-based EMC is a promising approach to achieve EMC in many environments, and also in the hospital, which is a complex and time-varying EM environment. Due to the new requirements for medical devices, the risk management aspect is even mandatory. In this paper, we discussed the factors that can cause EMI in hospital. To visualize the effect of multiple reflections, the EM field strength was measured with the help of an RSSI device both in free space and in reflecting the complex environment. These basic measurements hint that the distribution of the EM fields in free space, like an anechoic chamber, is different than in an enclosed hospital room, which behaves more like a semi-reverberant environment. The process for a risk-based approach in the military domain, the new Guide for the EMC Directive, and the standards and Medical Devices Regulations (MDR) were compared. An initial approach was developed for a hospital, as a complex EM environment, which implements the risk-based EMC approach to prevent EMI.

\section{REFERENCES}

[1] E. Ing, K. Armstrong, and S. Mieee, "Another EMC resource from EMC Standards EMC requirements in IEC60601-1-2 Taster The medical EMC requirements in IEC 60601-1-2, s 2007 and 2014 editions," vol. 44, no. 0, pp. 0-4.

[2] "March 2018 Guide for the EMCD ( Directive 2014 / 30 / EU )," no. March, pp. 1-67, 2018.

[3] C. Standard, "NEN-EN-IEC 60601-1-2," 2014.

[4] F. Leferink, "Risk-based vs Rule-based Electromagnetic Compatibility in Large Installations," 2018 IEEE 4th Glob. Electromagn. Compat. Conf. GEMCCon 2018, 2019,

[5] F. Leferink, J. van der Ven, H. Bergsma and B. van Leersum, "Risk based EMC for complex systems," 2017 XXXIInd General Assembly and Scientific Symposium of the International Union of Radio Science (URSI GASS), Montreal, QC, 2017,.

[6] V. Dafinescu, V. David, and I. Nica, "Medical devices electromagnetic interference due to radiated emissions in the hospital,” 2011 E-Health Bioeng. Conf. EHB 2011, , 2011.

[7] Seungmin Chung, Electromagnetic Interference of Wireless Local Area Network on Electrocardiogram Monitoring System: A case Report, 2013

[8]. A. Sathyanarayanan, A. Aggrwal and S. Chand, "Case study-MRI facility in hospital environment," 1995 International Conference on Electromagnetic Interference and Compatibility (INCEMIC), Madras, India, 1995, pp. 273-279

[9] D.D.J. Paus, F. Leferink "Universiteit Twente EMC-problematiek in de ambulancezorg na invoering TETRA ( C2000 )," Report 2000.

[10] J. L. Silberberg, "An FDA Perspective on Medical Device EMC and Wireless WED-PM-4," 2018 IEEE Symp. Electromagn. Compat. Signal Integr. Power Integrity, EMC, SI PI 2018, 2018,

[11] A. J. Trigano, A. Azoulay, M. Rochdi, and A. Campillo, "Electromagnetic interference of external pacemakers by walkietalkies and digital cellular phones: Experimental study," PACE Pacing Clin. Electrophysiol., vol. 22, no. 4 I, pp. 588-593, 1999,

[12] G. Calcagnini et al., "Electromagnetic interference to infusion pumps from GSM mobile phones," Annu. Int. Conf. IEEE Eng. Med. Biol. Proc., vol. 26 V, pp. 3515-3518, 2004,
[13] [online] Available https://www.medicaldesignbriefs.com/component/content/ aricle $/ \mathrm{mdb} /$ features/articles $/ 33060$

[14] Regulation (EU) 2017/745 [online] Available. https://eurlex.europa.eu/legal-content /EN/TXT/?uri= CELEX: 32017R0745

[15] N. J. LaSorte, Y. Burette, and H. H. Refai, "Experimental characterization of electromagnetic propagation of a hospital from 55-1950MHz," 2010 Asia-Pacific Symp. Electromagn. Compat. APEMC 2010, pp. 826-829, 2010,

[16] C. Kurnaz and T. Aygun, "Characterization of Indoor and Outdoor Electric Field Strength Levels at Hospitals," 2018 26th Telecommunications Forum (TELFOR), Belgrade, 2018, pp. 1-4

[17] H. Alnamir, "Study of Low Frequency Electromagnetic Interference Problems in Hospital Environment," 2019 11th Int. Symp. Adv. Top. Electr. Eng. ATEE 2019, pp. 1-5, 2019,

[18] R. Vogt-Ardatjew, U. Lundgren, S. F. Romero, and F. Leferink, "OnSite Radiated Emissions Measurements in Semireverberant Environments," IEEE Trans. Electromagn. Compat., vol. 59, no. 3, pp. 770-778, 2017,

[19] R. Vogt-ardatjew, T.T.M. Palstra "Electromagnetic fields in reverberant environments." Ph.D. dissertation, University of Twente 2017.https://ris.utwente.nl/ws/portalfiles/portal/18868825/Thesis_V ogt.pdf

[20] T. Hikage, T. Nojima, A. Y. Simba, and S. Watanabe, "Numerical estimation of EMI impact on implantable cardiac pacemakers in elevator using EMF distributions inside human body," 2010 IEEE Antennas and Propagation Society International Symposium, Toronto, ON, 2010, pp. 1-4,

[21] M. D. Hatagundi and G. Sadashivappa, "Wireless Communication in Medical Devices: Research Challenges," 2018 2nd International Conference on Trends in Electronics and Informatics (ICOEI), Tirunelveli, 2018, pp. 336-338,

[22] S.P.P. Jeunink, F. Leferink "Mathematics \& Computer Science Electromagnetic Sources in the Hospital Environment: Risks Analysis and Measures for Compatibility ( EMC )," Master Thesis University of Twente, 2017.

[23] G. Calcagnini, E. Mattei, F. Censi, M. Triventi, R. L. Sterzo, E. Marchetta, and P. Bartolini, "Electromagnetic compatibility of wlan adapters with life-supporting medical devices,"

[24] T N O Report, R. Hensbroek, M.Sc "96 Medical Apparatuses tested for interference by WLAN / WiFi signals," Area, 2007.

[25] E. Hanada and T. Kudou, "Managing the electromagnetic environment of hospital IoT systems," 2018 IEEE Int. Symp. Electromagn. Compat. 2018 IEEE Asia-Pacific Symp. Electromagn. Compat. EMC/APEMC 2018, pp. 940-943, 2018,

[26] K. Ishida, K. Suzuki, E. Hanada, and M. Hirose, "EMC of wireless medical telemeters and noise radiated from light emitting diode lamps," 2017 Int. Symp. Electromagn. Compat. - EMC Eur. 2017, EMC Eur. 2017, pp. 8-11, 2017,

[27] R. M. Nelson and H. Ji, "Electric and magnetic fields created by electrosurgical units," IEEE Trans. Electromagn. Compat., vol. 41, no. 1, pp. 55-64, 1999,

[28] M. Fernández Chimeno and F. Silva Martínez, "RFID systems in medical environment: EMC issues," 2010.

[29] L. Fu, Z. Yan, and G. Shi, "The electromagnetic environment assessment of nuclear magnetic resonance device in hospital," 2012 10th Int. Symp. Antennas, Propag. EM Theory, ISAPE 2012, pp. 1099-1102, 2012,

[30] R. Harris, "A Practical Approach to Risk Assessment and Risk Reduction Today's Learning Objectives," 2012. [online] Available 